Published in "American Journal of Physiology Regulatory, Integrative and Comparative Physiolology, 2010

doi: 10.1152/ajpregu.00404.2009" which should be cited to refer to this work.

\title{
Role of Mutation of the Circadian Clock Gene Per2 in Cardiovascular Circadian Rhythms
}

Ana Vukolic, Vladan Antic, Bruce N. Van Vliet, Zhihong Yang, Urs Albrecht and Jean-Pierre Montani

From the Department of Medicine/Physiology (A.V., V.A., Z.Y., J.P.M) and Biochemistry (U.A), University of Fribourg, Fribourg, Switzerland; and Memorial University of

Newfoundland (B.N.V.V), St. John's, Canada

Short title: Clock genes \& cardiovascular circadian rhythms

Total word count with Title, Abstract, References, Tables and Figure legends, but excluding Figures: 7’'107

Word count of abstract: 295

Number of Tables: 3

Number of Figures: 4

Correspondence to Jean-Pierre Montani, Department of Medicine/Division of Physiology, University of Fribourg, Chemin du Musée 5, CH-1700 Fribourg/Switzerland Phone +41-26-300-8591, Fax +41-26-300-9734. E-mail: jean-pierre.montani@unifr.ch 


\begin{abstract}
Alterations in the circadian blood pressure pattern are frequently observed in hypertension and lead to increased cardiovascular morbidity. However, there are no studies that have investigated a possible implication of the Period2 gene, a key component of the molecular circadian clock, on the circadian rhythms of blood pressure and heart rate. To address this question, we monitored blood pressure, heart rate and locomotor activity $24 \mathrm{~h}$ a day by telemetry in mice carrying a mutation in the Period2 gene and in wild type control mice. Under a standard 12h/12h light-dark cycle mutant mice showed a mild cardiovascular phenotype with an elevated $24 \mathrm{~h}$ heart rate, a decreased $24 \mathrm{~h}$ diastolic blood pressure and an attenuation of the dark-light difference in blood pressure and heart rate. Locomotor activity was similar in both groups and did not appear to explain the observed hemodynamic differences. When mice were placed under constant darkness during 8 consecutive days, wild type mice maintained $24 \mathrm{~h}$ rhythms whereas there was an apparent progressive loss of $24 \mathrm{~h}$ rhythm of blood pressure, heart rate and locomotor activity in mutant mice. However, a chisquare periodogram revealed that circadian rhythms were preserved under complete absence of any light cue, but with shorter periods by about $40 \mathrm{~min}$, leading to a cumulative phase shift towards earlier times of about $5 \mathrm{~h}$ and $20 \mathrm{~min}$ by the end of the $8^{\text {th }}$ day. When heart rate, mean arterial pressure and activity were recalculated according to the endogenous circadian periods of each individual mouse, the amplitudes of the circadian rhythms ("subjective night""subjective day" differences) were maintained for all variables studied. Our data show that mutation of the Period2 gene results in an attenuated dipping of blood pressure and heart rate during both light-dark cycles and constant darkness, and in shorter circadian periods during constant darkness.
\end{abstract}

Key words: circadian rhythm, clock genes, heart rate, blood pressure, telemetry 


\section{Introduction}

Circulatory demands change greatly over the day and night, and the ability to anticipate these changes is beneficial to the survival of the individual. Blood pressure (BP), heart rate (HR), cardiac output and stroke volume change rhythmically according to a 24-hour cycle, in humans being higher during the day than during the night $(20 ; 23)$. A number of studies provide evidence that the endogenous circadian oscillating system in the suprachiasmatic nucleus $(\mathrm{SCN})$ plays an important role in cardiovascular control as SCN lesion eliminates $24-\mathrm{h}$ rhythm of BP and HR in experimental animals $(15 ; 36 ; 37)$.

Perturbations of circadian rhythms may contribute to cardiovascular pathology. Disturbances at the level of central circadian clock are suggested in human hypertension (12; 13) as well as in some animal models of hypertension $(9 ; 10)$. Hypertensive patients whose average night-time BP fails to 'dip' relative to their average day-time BP by at least $10 \%$ ('non-dippers') have a significantly greater frequency of left ventricular hypertrophy, microalbuminuria, and stroke $(14 ; 29 ; 41)$ than do individuals whose BP drops during the night by $10 \%$ or more.

In mammals, circadian rhythms of physiology, biochemistry and behavior are orchestrated by a master clock situated in the SCN of the hypothalamus (49). The endogenously generated circadian rhythm maintains a period of approximately 24 hours even in the absence of environmental cues (33). Molecular circadian regulation engages a transcription-translation feedback loop comprising clock proteins, CLOCK, BMAL1, PER(s) and $\mathrm{CRY}(\mathrm{s})(4 ; 8 ; 40)$. Circadian expression of clock genes has also been demonstrated throughout the body, such as in the heart (38), liver (19), adrenal cortex (6), or kidney and other organs (5). 
The Period 2 (Per2) gene is a key molecular component in controlling mammalian circadian rhythms (2) at the levels of gene expression, physiology and pathology. A robust circadian fluctuation of Per2 mRNA expression was discovered not only in the SCN but also in many peripheral tissues. The Per genes are crucial for circadian phase shifts by delaying the clock phase and regulates expression of another clock gene (Bmal1) in a positive manner. Furthermore, alterations in Per2 genes affect many biochemical processes, showing the multiple facets of the Per2 gene (reviewed by (3)). The behavioral phenotype of Per2 mutants in constant darkness is characterized by short period rhythms and a progressive loss of the circadian rhythm of wheel-running locomotor activity (51). In addition, a lack of food anticipation (seen through the lack of food-anticipatory activity and lack of body temperature changes in anticipation of food) is specifically associated with a mutation of Per2, demonstrating the critical involvement of this gene in the anticipation of meal time (11). Several authors have already suggested potential involvement of Per2 gene in regulation of cardiovascular system. It was shown that the protein encoded by Per2 gene (PER2) is expressed in circadian manner in aortic smooth muscle cells (32) and that disturbed diurnal rhythm alters Per2 gene expression both in SCN and the heart of a mouse model of pressure overload cardiac hypertrophy (22). In addition, Per2 mutant mice display altered vascular endothelial function (48). However, there are no studies that have directly analyzed the role of the Per2 gene on the circadian control of BP and HR. To address this question, we analyzed in Per2 mutant mice and wild type control mice the circadian rhythms of BP, HR and locomotor activity, both in the presence and in the absence of the most powerful external cue, i.e. the light-dark (LD) cycle. 


\section{Methods}

\section{Animals}

Mutant mice (mPer2Brdm1), designated here as Per 2 mutants, were obtained from Zheng et al. (51) and propagated in our own facility. In Per2 mutants PER2 protein is lacking the carboxy-terminal half of the PAS B and the entire PAC domain, resulting in a significantly shortened protein that lacks possible dimerisation sites (reviewed by (3)). All experiments were performed on adult 3-4 months old wild-type (WT) (129 SvEvBrd X C57B16-Ty) and Per2 mutant mice. In this study we used only male mice to avoid estrousrelated variations in circadian pattern of $\mathrm{BP}, \mathrm{HR}$ and locomotor activity, which have been described in female rats $(43 ; 50)$ and mice $(17)$. Homozygous mutants as well as wild types were kept on an artificial 12h/12h LD cycle with lights off from 18.00 to $6.00 \mathrm{~h}$. Temperature and humidity were kept stable at $22 \pm 1^{\circ} \mathrm{C}$ and $55 \pm 5 \%$, respectively. Animals were housed individually and provided with normal chow (Diet 3433, Provimi-Kliba, Switzerland) and water ad libitum. The animal experimental protocol was approved by the Ethical Committee of State Veterinary Office of Fribourg, Switzerland.

\section{Blood pressure telemetry}

All surgical procedures were performed using aseptic techniques, premedication with atropine sulphate $0.03 \mathrm{mg} / \mathrm{kg}+$ carprofen $10 \mathrm{mg} / \mathrm{kg}$ i.m. and under general anesthesia with ketamine $90 \mathrm{mg} / \mathrm{kg}+$ xylazine $10 \mathrm{mg} / \mathrm{kg}$ i.p. All mice were instrumented with an arterial catheter connected to an implantable transducer (model TA11PA-C10, Data Sciences International, St. Paul, MN, U.S.A.) to monitor arterial pressure and HR $24 \mathrm{~h}$ a day by telemetry in unrestrained mice. The tip of the telemeter catheter was inserted into the aortic arch via the left common carotid artery as $\underset{5}{\operatorname{sescribed}}$ previously (46). After completion of the 
procedure, the mice were housed in individual standard mouse cages $(23 \mathrm{~cm} \times 17 \mathrm{~cm} \times 14 \mathrm{~cm}$ high, Indulab AG, Switzerland) for continuous BP and HR measurement and monitoring of daily food intake. At least 12 days were given to animals to recover from surgery and for BP and HR to stabilize.

Animal cages were equipped with one RPC-1 plastic receiver (Data Sciences Int.) fixed on the bottom of the cage and connected to an analog output device (R11-CPA), allowing for the continuous recording of the analog pulsatile arterial pressure signal. The signal was then fed to a 12-bit analog-to-digital converter, sampled at $1 \mathrm{kHz}$, and processed using customized algorithms for beat-to-beat analysis (24). Locomotor activity was estimated from the number of counts provided by the receiver, reflecting movements of the animal above a certain threshold. Each telemeter transducer was calibrated before implantation, as well as after explantation. Corrections for offset drift were not needed because the drift was less than $2 \mathrm{mmHg}$ in all mice.

\section{Experimental protocol}

In all animals (27 Per2 mutants and 30 WT control mice) we studied basal HR, BP and locomotor activity under the standard $12 \mathrm{~h} / 12 \mathrm{~h}$ LD cycle during two consecutive days (protocol 1). In a subset of animals (9 Per2 mutants and 6 WT mice), we studied the effects of constant darkness (DD) on circadian periods of cardiovascular parameters (protocol 2). After 2 days of control under 12h/12h LD cycle in a customized ventilated chamber, mice were submitted to 8 consecutive days of DD, with no exposure to external cues (noise or light) and with enough food and water to sustain the 8 days of DD. This was followed by a 3day recovery under regular $12 \mathrm{~h} / 12 \mathrm{~h} \mathrm{LD}$ cycle. 


\section{Measurements under constant darkness}

Comparison of six methods for the determination of the period of circadian rhythm (Fourier analysis, autocorrelation, chi-square periodogram, linear regression of onset, interonset averaging and acrophase counting ) revealed that Fourier analysis and chi-square periodogram were superior to the other methods in the analysis of more complex waveforms (which more closely resemble actual circadian rhythms) (30). Furthermore, when random noise was inserted into the original data set, the chi-square periodogram was more tolerant to low signal-to-noise ratio than Fourier analysis. Thus, we used the chi-square periodogram $(30 ; 31)$ to calculate circadian periods of mean arterial pressure (MAP), HR and activity for the 8 consecutive days under DD. A resolution of 10 minutes (bin size) under DD was selected as input data, which give a $0.17 \mathrm{~h}$ resolution of the computed periods.

First, the daily 24h-records for both LD and DD experimental days were divided in two equal periods of $12 \mathrm{~h}$, from 18.00-06.00 h (ZT12 to ZT24, representing "night") and from 06.00-18.00 h (ZT0 to ZT12, representing "day") to compute daily mean "night" minus mean "day" differences, defined here as "night" - "day" difference. ZT stands for "Zeitgeber time", i.e., time defined relative to experimental LD cycle where the onset of light defines the Zeitgeber time 0 (ZT0) and the onset of dark defines Zeitgeber time 12 (ZT12). Then, the circadian periods of HR, MAP and activity under DD, obtained by the chi-square periodogram, were used to calculate the "subjective" day/night cycle of each individual mouse and divided in two equal periods corresponding to "subjective day" (CT0 to CT12) and "subjective night" (CT12 to CT24) for these parameters. Again, we computed mean "subjective night" minus mean "subjective day" differences, defined here as "subjective night"- "subjective day" difference and plotted over CT, where CT stands for "Circadian 
time" i.e. standard of time based on the free-running period of a rhythm where the onset of activity defines circadian time 12 (CT12).

A second analysis program (Cosinor periodogram by R. Refinetti, http://www.circadian.org/softwar.html) was used to compare the circadian periods between LD and DD. This was necessary because the chi-square periodogram, although the most reliable tool when a sufficient number of input days is available, was not able to compute the period of circadian rhythms for two days of LD cycle.

Finally, to compute the acrophase (time at which the peak of the rhythm is reached) and the threshold (time at which the trough of the rhythm is reached), we used a fitted cosine wave procedure (Acro program by R. Refinetti, http://www.circadian.org/softwar.html ) for both LD and each of the 8 days under DD. The times of acrophase and threshold are given in hours from midnight.

\section{Statistical analysis}

All data are presented as mean \pm standard error. In protocol 1 an unpaired t-test was used to compare data between Per2 mutants and WT mice. In protocol 2 daily changes from control baseline values were analyzed with an analysis of variance for repeated measurements, followed by a multiple comparisons Dunnett's t test. Comparisons between Per2 mutants and WT mice were done with an unpaired t-test, with Bonferroni correction in the case of multiple comparisons. All statistics were calculated using the GraphPad InStat statistical program, version 3.01. A value of $\mathrm{p}<0.05$ was used for statistical significance. 


\section{Results}

\section{Basal hemodynamic values under 12h/12h LD cycle (protocol 1)}

Basal values collected during two consecutive days of $12 \mathrm{~h} / 12 \mathrm{~h} \mathrm{LD}$ cycle (protocol 1) for MAP, systolic and diastolic pressures (SBP and DBP), HR and locomotor activity are given in Table 1. Data are presented as $24 \mathrm{~h}$ averages as well as separately for the light and the dark periods. 24-hour HR was higher in Per2 mutant mice than in WT mice. BP tended to be lower in mutant mice, but this was significant only for DBP. When analyzing separately light and dark periods, the increase in HR was confined to the light period whereas the decrease in DBP was significant only during the dark period. Mean locomotor activity (expressed as number of activity signals received per 30 seconds) was similar in both types of mice.

In order to assess the influence of locomotor activity on hemodynamic parameters we reanalyzed the data by separating the 30 -second periods in which the animal was moving (active periods) from the periods in which no apparent movements were detected (inactive periods), as shown in Table 2. In fact, the higher HR in Per2 mutants seemed more related to the resting state as the difference in HR between WT and mutant mice was greater during periods of inactivity (see Table 2). On the other hand, DBP tended to be lower in Per2 mutants when the animals were active. Although mean locomotor activity was similar in both types of mice (Table 1), Per2 mutants showed a slightly higher percentage of time spent active during the light period (Table 2).

Differences between the 12h-dark period and the 12h-light period (dark-light difference) were computed and are reported in Figure 1. Per2 mutants showed significant decreases in dark-light differences for MAP (panel A, p = 0.002) and HR (panel B, p = 
0.0004), but not of locomotor activity (panel C). Dark-light differences were also significantly smaller for SBP and DBP $(\mathrm{P}=0.01$ and $\mathrm{P}<0.0001$, respectively; data not shown $)$.

\section{Hemodynamic analysis under constant darkness (protocol 2)}

Figure 2 illustrates the calculated circadian periods by chi-square periodogram in WT and Per2 mutant mice during constant darkness when all 8 consecutive days were pooled for a single analysis of the duration of the average circadian period. In WT mice circadian periods were fully preserved and amounted to about $24 \mathrm{~h}$ for MAP $(23.94 \pm 0.04 \mathrm{~h}), \mathrm{HR}(24.00 \pm 0.07$ h) and activity $(24.05 \pm 0.09$ h). Per2 mutants also showed rhythmicity under constant darkness, but with significantly shorter periods for MAP $(23.31 \pm 0.12 \mathrm{~h}), \mathrm{HR}(23.26 \pm 0.08$ h) and activity $(23.33 \pm 0.19 \mathrm{~h})$ and a wider distribution of individual values.

The calculated circadian periods by cosinor periodogram in WT and Per2 mutant mice both during LD (pool of two consecutive days) and during DD (pool of 8 consecutive days) are shown in Table 3. Under LD there were no differences in the circadian periods between WT mice and Per2 mutants for all three variables studied (MAP, HR and activity). During DD, however, Per2 mutants had significantly shorter circadian periods of MAP, HR and activity comparing to WT counterparts. Comparing DD versus LD, WT mice showed similar circadian periods whereas Per2 mutants had shorter circadian periods (but significant only for MAP and HR).

Figure 3 (left panels A1, B1 and C1) shows the 12h/12h "night"-“day" difference for MAP, HR and locomotor activity during the two control days under 12h/12h LD cycle, during the 8 days of constant darkness (DD) and during the first 3 days of recovery under $12 \mathrm{~h} / 12 \mathrm{~h}$ LD cycle. All data are plotted over ZT time, i.e. relative to experimental LD cycle. During constant darkness, WT mice showed a preserved 12h/12h "night”-“day" difference for MAP, HR and locomotor activity during all 8 days of DD. In contrast, Per 2 mutant mice showed a 
progressive alteration of the $12 \mathrm{~h} / 12 \mathrm{~h}$ "night"-“day" difference for all 3 variables studied (panels A1, B1 and C1). When the animals were returned under a normal 12h/12h LD cycle (3 days of recovery), WT mice maintained their stable "night"-"day" differences and Per2 mutant mice showed a progressive return of the "night"-"day" differences toward basal control values.

Because of the shorter circadian periods computed in Per2 mutants, "night"-“day" differences under DD were recalculated on the basis of the endogenous (i.e. free-running) circadian periods of MAP, HR and activity obtained for each individual mouse by the chisquare periodogram, and are expressed as "subjective night" - "subjective day" difference. As shown in Figure 3 (right panels A2, B2 and C2), the time axis represents now, not a 24hour time based on $12 \mathrm{~h} / 12 \mathrm{~h}$ LD cycle (ZT), but CT. Thus, the beginning of the "subjective day" is at CT0, and the beginning of the "subjective night" is at CT12. Recalculated data showed that Per2 mutant mice were able to conserve circadian rhythms of all three variables under constant darkness. As circadian periods in mutant mice under DD were significantly shorter than 24 hours, the mice were already in "subjective day" for about $5 \mathrm{~h}$ and $20 \mathrm{~min}$ at the beginning of the light period upon return to normal 12h/12h LD after 8 days under DD. Thus, it was not possible to calculate the "night"-"day" difference according to the circadian periods during the 3 days of recovery. The two control days under $12 \mathrm{~h} / 12 \mathrm{~h} \mathrm{LD}$ cycle are the same for all panels, as here $\mathrm{CT}=\mathrm{ZT}$.

Figure 4 shows the acrophase and the threshold of the rhythms for MAP (panel A), HR (panel B) and activity (panel C). Data are plotted in hours from midnight during LD (as average for 2 control days) and for each of the 8 days under DD. In WT mice both the acrophase and the threshold of the rhythms during all 8 days of DD remained similar to LD value. In contrast, Per2 mutants showed a progressive shift from LD that by the $8^{\text {th }}$ day led to a cumulative advanced time shift of $4.9 \mathrm{~h}$ for MAP threshold (from $14.2 \mathrm{~h}$ to $9.3 \mathrm{~h}$ ) and $5.1 \mathrm{~h}$ for 
MAP acrophase (from $22.6 \mathrm{~h}$ to $17.5 \mathrm{~h}$ ). Similar results were found for HR threshold (5.4h advanced shift, from $12.5 \mathrm{~h}$ to $7.1 \mathrm{~h}$ ) and HR acrophase (5.8h advanced shift, from $21.1 \mathrm{~h}$ to $15.3 \mathrm{~h}$ ), as well as for activity threshold (5.8h advanced shift, from $13.4 \mathrm{~h}$ to $7.6 \mathrm{~h}$ ) and activity acrophase (6h advanced shift, from $21.9 \mathrm{~h}$ to $15.9 \mathrm{~h}$ ).

\section{Discussion}

The present study focused on the possible role of the clock gene Per2 in the circadian control of BP and HR, as this gene is considered to be a key component of the molecular circadian clock $(1 ; 16)$. The most important findings of our study are that mutation of the Per2 gene results in an attenuation of the dark-light difference in BP and HR under LD cycle and shorter circadian periods of BP, HR and locomotor activity under constant darkness.

Under a standard LD cycle, Per2 mutant mice showed a cardiovascular phenotype characterized by a higher HR during the light period, a lower DBP during the dark period, and a decreased dark-light difference in BP and HR. Since locomotor activity may represent a very important confounding factor in the analysis of BP and HR, particularly in mice $(46 ; 47)$, we also compared hemodynamic values during periods of activity and inactivity, as shown in Table 2. Intriguingly, the increase in HR during the light period and the decrease in DBP during the dark period were found during both active and inactive periods, suggesting that locomotor activity is not the major determinant of the phenotype difference between Per2 mutant and WT mice. Furthermore, the average intensity of locomotor activity in Per2 mutants was not statistically different from that of WT mice under LD conditions (see Table 1) despite a slightly greater percentage of time spent in active periods during the light period (see Table 2). These data are consistent with previous observations that Per2 mutant animals do not differ in the amount of wheel running activity from their WT counterparts (27). 
In addition, the Per 2 mutant mice have a tendency for lower BP, which was significant only for DBP during the dark period. This was unexpected as we previously described that isolated vessels of Per 2 mutant mice display altered vascular endothelial function due to a decreased production of nitric oxide and vasodilatory prostaglandins as well as an increased release of COX-1-derived vasoconstrictors (48). This could suggest that BP regulation is not dominated by peripheral clocks in blood vessels.

Another interesting finding under LD cycle was an attenuation of the dark-light difference in Per2 mutants that was statistically significant for MAP and HR, but not for locomotor activity (Figure 1). We can only speculate on the causes of the attenuated dark-like difference. A possible explanation is an autonomic imbalance with an attenuation of sympathetic inhibition during the light period, consistent with the higher HR observed during this period. In fact, the autonomous nervous system is probably the most important mechanism by which the SCN controls the circadian rhythm of BP and HR $(7 ; 38 ; 44)$. In humans, deregulation of the circadian control of the autonomic nervous system may lead to non-dipping pattern of blood pressure associated with and potential target-organ damage in hypertensive patients $(29 ; 41)$ and, more recently published, in normotensive subjects $(42)$.

As shown in Table 3, a 24h-rhythm was preserved during LD in Per2 mutants and did not differ from WT mice. To assess free-running circadian rhythms in the absence of the most powerful external cue (Zeitgeber), i.e. the LD cycle, it is important to study animals under constant conditions. It is known that Per2 mutants under constant darkness have a shorter circadian rhythm period of wheel-running activity than their WT counterparts (51). However, free-running circadian rhythm periods of BP and HR in Per2 mutants have not been reported. When both types of mice were placed under constant darkness during 8 consecutive days, WT mice conserved circadian rhythm amplitudes whereas there was an apparent progressive attenuation of BP, HR and locomotor activity rhythm amplitudes in mutant mice. However, 
based on both chi-square and cosinor periodogram analyses, a circadian period for all 3 variables studied (MAP, HR and locomotor activity) was observed in Per2 mutant mice, but was significantly shorter than in WT counterparts, as shown in Figure 2 and Table 3. This shortening could be due to a defect in the central clock. Indeed, expression of Per2 in SCN of WT controls peaks at around ZT12 and in Per2 mutants at around ZT10 (51). This phase advance of Per 2 expression in $\mathrm{SCN}$ could be responsible for the phase advance in daily rhythms observed in Per2 mutants. Furthermore, patients with a mutation in the binding site for casein kinase 1 in the Per2 gene show a familial advanced sleep phase syndrome (45). A daily shortening of circadian rhythms observed in our experiments would lead to a cumulative advanced phase shift of the "subjective" day/night cycle by the $8^{\text {th }}$ day of DD, explaining the progressive attenuation in the circadian amplitudes seen in panels A1 to $\mathrm{C} 1$ of Figure 3 when data are plotted based on ZT. When data were plotted using CT instead of ZT, Per2 mutants cannot be considered to be arrhythmic, at least not for the first 8 days under DD, based on the preservation of the amplitude of the circadian rhythms of MAP, HR and activity (panels A2 to $\mathrm{C} 2$ of Figure 3). On the other hand, WT mice maintained a 24h rhythm even under the complete absence of any light cue. This finding is supported by the results seen in Figure 4, showing that the acrophase and the threshold of the rhythms in the WT mice under DD appeared at the same time of the day as in the LD period. In contrast, Per2 mutants showed a progressive advanced shift of the both acrophase and the threshold, leading to an average cumulative shift of between 5 to $6 \mathrm{~h}$ by the end of the $8^{\text {th }}$ day. This advanced shift in acrophase and threshold is consistent with the about $5 \mathrm{~h}$ and 20 minutes phase advanced shift obtained with the chi-square periodogram. Moreover, the shortening of the rhythms seems to occur from the very first day of DD based on acrophase and threshold values in Figure 4.

The shorter periods of BP and HR in Per2 mutants are probably not linked to the shorter period of activity. First, a loss of the circadian rhythm of wheel-running activity in 
Per2 mutants can occur very early (range between 2 and 18 days) upon introduction of DD (51). Second, two Per2 mutant mice in our study did not show a rhythm for locomotor activity with the chi-square periodogram, but had rhythms for MAP and HR during 8 days of DD. This could imply that circadian locomotor activity rhythm is decoupled from MAP and HR rhythms in Per2 mutant mice, and probably the first one to be lost under constant conditions. The maintenance of HR circadian rhythms even when activity rhythms were lost is consistent with the finding in humans that the circadian rhythm in HR could be independent of the activity rhythm $(18 ; 39)$. WT mice circadian period lengths under DD were very consistent for all animals. As shown in Figure 2, the highest value for MAP circadian period was $24.00 \mathrm{~h}$ and the lowest value was $23.73 \mathrm{~h}$. A similar narrow range was found for HR (highest value $24.33 \mathrm{~h}$, lowest value $23.83 \mathrm{~h}$ ) and for activity (highest value $24.5 \mathrm{~h}$, lowest value 23.83h). In contrast, Per 2 mutant mice showed a wide distribution of the same parameters with MAP period ranging from $22.70 \mathrm{~h}$ to $23.97 \mathrm{~h}$, HR period ranging from $23.00 \mathrm{~h}$ to $23.62 \mathrm{~h}$, and locomotor activity period ranging from $22.50 \mathrm{~h}$ to $24 \mathrm{~h} 00$. This lack of uniformity among period lengths in Per2 mutants further enhances the alteration of "night"-“day" differences under DD when a $12 \mathrm{~h} / 12 \mathrm{~h}$ ZT cycle is used to calculate the data.

Substantial evidence has accumulated that the SCN orchestrates circadian oscillations in sympathetic and parasympathetic activity, and alterations in its function may thereby affect the cardiovascular system. Deregulation of the circadian control of the endocrine system, mainly of the reactivity of the hypothalamic-pituitary-adrenal axis, may further impair the adaptation of the cardiovascular system to external challenges. Moreover, alterations in the peripheral circadian clock mechanisms, particularly in the heart, kidney and vascular smooth muscle cells may compromise the harmonious regulation of cardiovascular function and jeopardize its ability to adapt to circadian changes in exogenous demands. However, their quantitative contribution to the Per2 phenotype is not clear. 


\section{Perspectives}

Epidemiological evidence shows that cardiovascular events occur more frequently at certain periods of the day in humans. A number of cardiovascular pathologies such as myocardial ischemia (35), myocardial infarction (26), sudden cardiac death (25) and ischemic stroke (21), show a diurnal pattern. Furthermore, circadian rhythm disruption is found commonly in patients with sleep apnea (28), transmeridian flight crews and shift workers (34), all of whom show higher than average prevalence of cardiovascular diseases. A better understanding in the control of cardiovascular circadian rhythms may lead to health benefits. Our present results suggest that the Per2 gene provides a significant contribution to cardiovascular circadian rhythms.

\section{Source of Funding}

This study was supported in part by the Swiss National Science Foundation.

\section{Conflict of Interest}

The authors declare no conflict of interest. 
1. Albrecht $\mathbf{U}$. Invited review: regulation of mammalian circadian clock genes. $J$ Appl Physiol 92: 1348-1355, 2002.

2. Albrecht U. Per2 has time on its side. Nat Chem Biol 3: 139-140, 2007.

3. Albrecht U, Bordon A, Schmutz I and Ripperger J. The multiple facets of Per2. Cold Spring Harb Symp Quant Biol 72: 95-104, 2007.

4. Albrecht $\mathbf{U}$ and Eichele G. The mammalian circadian clock. Curr Opin Genet Dev 13: 271-277, 2003.

5. Balsalobre A. Clock genes in mammalian peripheral tissues. Cell Tissue Res 309: 193199, 2002.

6. Buijs RM, Wortel J, Van Heerikhuize JJ, Feenstra MG, Ter Horst GJ, Romijn HJ and Kalsbeek A. Anatomical and functional demonstration of a multisynaptic suprachiasmatic nucleus adrenal (cortex) pathway. Eur J Neurosci 11: 1535-1544, 1999.

7. Cui LN, Coderre E and Renaud LP. Glutamate and GABA mediate suprachiasmatic nucleus inputs to spinal-projecting paraventricular neurons. Am J Physiol Regul Integr Comp Physiol 281: R1283-R1289, 2001.

8. Dunlap JC, Loros JJ, Liu Y and Crosthwaite SK. Eukaryotic circadian systems: cycles in common. Genes Cells 4: 1-10, 1999.

9. Eilam R, Malach R, Bergmann F and Segal M. Hypertension induced by hypothalamic transplantation from genetically hypertensive to normotensive rats. $J$ Neurosci 11: 401-411, 1991.

10. Eilam R, Malach R and Segal M. Selective elimination of hypothalamic neurons by grafted hypertension-inducing neural tissue. J Neurosci 14: 4891-4902, 1994.

11. Feillet CA, Ripperger JA, Magnone MC, Dulloo A, Albrecht $U$ and Challet E. Lack of food anticipation in Per2 mutant mice ${ }_{17}$ Curr Biol 16: 2016-2022, 2006. 
12. Goncharuk VD, van Heerikhuize J, Dai JP, Swaab DF and Buijs RM. Neuropeptide changes in the suprachiasmatic nucleus in primary hypertension indicate functional impairment of the biological clock. J Comp Neurol 431: 320-330, 2001.

13. Goncharuk VD, van Heerikhuize J, Swaab DF and Buijs RM. Paraventricular nucleus of the human hypothalamus in primary hypertension: activation of corticotropin-releasing hormone neurons. J Comp Neurol 443: 321-331, 2002.

14. Imai Y, Hozawa A, Ohkubo T, Tsuji I, Yamaguchi J, Matsubara M, Michimata M, Hashimoto J, Fujiwara T, Nagai K, Kitaoka H, Satoh H and Hisamichi S.

Predictive values of automated blood pressure measurement: what can we learn from the Japanese population - the Ohasama study. Blood Press Monit 6: 335-339, 2001.

15. Janssen BJ, Tyssen CM, Duindam H and Rietveld WJ. Suprachiasmatic lesions eliminate 24-h blood pressure variability in rats. Physiol Behav 55: 307-311, 1994.

16. King DP and Takahashi JS. Molecular genetics of circadian rhythms in mammals. Annu Rev Neurosci 23: 713-742, 2000.

17. Kopp C, Ressel V, Wigger $\mathbf{E}$ and Tobler I. Influence of estrus cycle and ageing on activity patterns in two inbred mouse strains. Behav Brain Res 167: 165-174, 2006.

18. Krauchi K and Wirz-Justice A. Circadian rhythm of heat production, heart rate, and skin and core temperature under unmasking conditions in men. Am J Physiol 267: R819R829, 1994.

19. la Fleur SE, Kalsbeek A, Wortel J and Buijs RM. Polysynaptic neural pathways between the hypothalamus, including the suprachiasmatic nucleus, and the liver. Brain Res 871: 50-56, 2000.

20. Lemmer B. Cardiovascular chronobiology and chronopharmacology. In: Biologic Rhythms in Clinical and Laboratory Medicine, edited by Touitou Y and Haus E. Heidelberg, Germany: Springer Verlag, 1992, p. 418-427.

21. Marler JR, Price TR, Clark GL, Muller JE, Robertson T, Mohr JP, Hier DB, Wolf PA, Caplan LR and Foulkes MA. Morning increase in onset of ischemic stroke. Stroke 20: 473-476, 1989. 
22. Martino TA, Tata N, Belsham DD, Chalmers J, Straume M, Lee P, Pribiag H, Khaper N, Liu PP, Dawood F, Backx PH, Ralph MR and Sole MJ. Disturbed diurnal rhythm alters gene expression and exacerbates cardiovascular disease with rescue by resynchronization. Hypertension 49: 1104-1113, 2007.

23. Millar-Craig MW, Bishop CN and Raftery EB. Circadian variation of blood-pressure. Lancet 1: 795-797, 1978.

24. Montani JP, Mizelle HL, Van Vliet BN and Adair TH. Advantages of continuous measurement of cardiac output 24 h a day. Am J Physiol 269: H696-H703, 1995.

25. Muller JE, Ludmer PL, Willich SN, Tofler GH, Aylmer G, Klangos I and Stone PH. Circadian variation in the frequency of sudden cardiac death. Circulation 75: 131138, 1987.

26. Muller JE, Stone PH, Turi ZG, Rutherford JD, Czeisler CA, Parker C, Poole WK, Passamani E, Roberts R, Robertson $\mathbf{T}$ et al. Circadian variation in the frequency of onset of acute myocardial infarction. N Engl J Med 313: 1315-1322, 1985.

27. Oster H, Yasui A, van der Horst GT and Albrecht U. Disruption of mCry2 restores circadian rhythmicity in mPer2 mutant mice. Genes Dev 16: 2633-2638, 2002.

28. Pankow W, Nabe B, Lies A, Becker H, Kohler U, Kohl FV and Lohmann FW. Influence of sleep apnea on 24-hour blood pressure. Chest 112: 1253-1258, 1997.

29. Pickering TG and Kario K. Nocturnal non-dipping: what does it augur? Curr Opin Nephrol Hypertens 10: 611-616, 2001.

30. Refinetti R. Laboratory instrumentation and computing: comparison of six methods for the determination of the period of circadian rhythms. Physiol Behav 54: 869-875, 1993.

31. Refinetti R. Non-stationary time series and the robustness of circadian rhythms. $J$ Theor Biol 227: 571-581, 2004.

32. Reilly DF, Curtis AM, Cheng Y, Westgate EJ, Rudic RD, Paschos G, Morris J, Ouyang M, Thomas SA and FitzGerald GA. Peripheral circadian clock rhythmicity is retained in the absence of adrenergic signaling. Arterioscler Thromb Vasc Biol 28: 121126, 2008. 
33. Reppert SM and Weaver DR. Molecular analysis of mammalian circadian rhythms. Annu Rev Physiol 63: 647-676, 2001.

34. Richardson GS. The human circadian system in normal and disordered sleep. J Clin Psychiatry 66 Suppl 9: 3-9, 2005.

35. Rocco MB, Barry J, Campbell S, Nabel E, Cook EF, Goldman L and Selwyn AP. Circadian variation of transient myocardial ischemia in patients with coronary artery disease. Circulation 75: 395-400, 1987.

36. Saleh MA and Winget CM. Effect of suprachiasmatic lesions on diurnal heart rate rhythm in the rat. Physiol Behav 19: 561-564, 1977.

37. Sano H, Hayashi H, Makino M, Takezawa H, Hirai M, Saito H and Ebihara S. Effects of suprachiasmatic lesions on circadian rhythms of blood pressure, heart rate and locomotor activity in the rat. Jpn Circ J 59: 565-573, 1995.

38. Scheer FA, Ter Horst GJ, van Der Vliet J and Buijs RM. Physiological and anatomic evidence for regulation of the heart by suprachiasmatic nucleus in rats. Am J Physiol Heart Circ Physiol 280: H1391-H1399, 2001.

39. Scheer FA, Van Doornen LJ and Buijs RM. Light and diurnal cycle affect human heart rate: possible role for the circadian pacemaker. J Biol Rhythms 14: 202-212, 1999.

40. Shearman LP, Sriram S, Weaver DR, Maywood ES, Chaves I, Zheng B, Kume K, Lee CC, van der Horst GT, Hastings MH and Reppert SM. Interacting molecular loops in the mammalian circadian clock. Science 288: 1013-1019, 2000.

41. Shimada K and Kario K. Altered circadian rhythm of blood pressure and cerebrovascular damage. Blood Press Monit 2: 333-338, 1997.

42. Soylu A, Yazici M, Duzenli MA, Tokac M, Ozdemir K and Gok H. Relation between abnormalities in circadian blood pressure rhythm and target organ damage in normotensives. Circ J 73: 899-904, 2009.

43. Takezawa H, Hayashi H, Sano H, Saito $\mathbf{H}$ and Ebihara S. Circadian and estrous cycle-dependent variations in blood pressure and heart rate in female rats. Am J Physiol 267: R1250-R1256, 1994. 
44. Ter Horst GJ and Postema F. Forebrain parasympathetic control of heart activity: retrograde transneuronal viral labeling in rats. Am J Physiol 273: H2926-H2930, 1997.

45. Toh KL, Jones CR, He Y, Eide EJ, Hinz WA, Virshup DM, Ptacek LJ and Fu YH. An hPer2 phosphorylation site mutation in familial advanced sleep phase syndrome. Science 291: 1040-1043, 2001.

46. Van Vliet BN, Chafe LL and Montani JP. Characteristics of $24 \mathrm{~h}$ telemetered blood pressure in eNOS-knockout and C57Bl/6J control mice. J Physiol 549: 313-325, 2003.

47. Van Vliet BN, McGuire J, Chafe L, Leonard A, Joshi A and Montani JP. Phenotyping the level of blood pressure by telemetry in mice. Clin Exp Pharmacol Physiol 33: 1007-1015, 2006.

48. Viswambharan H, Carvas JM, Antic V, Marecic A, Jud C, Zaugg CE, Ming XF, Montani JP, Albrecht $\mathbf{U}$ and Yang Z. Mutation of the circadian clock gene Per2 alters vascular endothelial function. Circulation 115: 2188-2195, 2007.

49. Weaver DR. The suprachiasmatic nucleus: a 25-year retrospective. J Biol Rhythms 13: 100-112, 1998.

50. Wollnik F and Turek FW. Estrous correlated modulations of circadian and ultradian wheel-running activity rhythms in LEW/Ztm rats. Physiol Behav 43: 389-396, 1988.

51. Zheng B, Larkin DW, Albrecht U, Sun ZS, Sage M, Eichele G, Lee CC and Bradley A. The mPer2 gene encodes a functional component of the mammalian circadian clock. Nature 400: 169-173, 1999. 
Table 1. Summary of basal hemodynamic values and locomotor activity acquired during $12 \mathrm{~h} / 12 \mathrm{~h}$ light-dark cycle in Per 2 mutants and wild type control mice.

\begin{tabular}{|c|c|c|c|c|}
\hline \multirow[t]{2}{*}{ Period } & & Wild type & Per 2 mutants & P value \\
\hline & & $(\mathbf{n}=\mathbf{3 0})$ & $(n=27)$ & \\
\hline \multirow[t]{5}{*}{$24 \mathrm{~h}$ values } & HR (bpm) & $520 \pm 5$ & $540 \pm 5$ & 0.007 \\
\hline & $\mathrm{SBP}(\mathrm{mmHg})$ & $128.7 \pm 1.3$ & $127.2 \pm 5$ & ns \\
\hline & MAP (mmHg) & $116.0 \pm 1.1$ & $113.8 \pm 1.3$ & ns \\
\hline & DBP (mmHg) & $104.3 \pm 1.2$ & $100.6 \pm 1.4$ & 0.05 \\
\hline & Mean activity (a.u./30s) & $2.0 \pm 0.2$ & $1.9 \pm 0.1$ & ns \\
\hline \multirow[t]{5}{*}{ Light period } & HR (bpm) & $497 \pm 5$ & $529 \pm 5$ & $<0.0001$ \\
\hline & SBP (mmHg) & $123.6 \pm 1.2$ & $123.3 \pm 1.7$ & ns \\
\hline & MAP (mmHg) & $110.9 \pm 1.1$ & $110.1 \pm 1.4$ & ns \\
\hline & DBP (mmHg) & $99.1 \pm 1.2$ & $97.1 \pm 1.5$ & ns \\
\hline & Mean activity (a.u./30s) & $0.9 \pm 0.1$ & $1.1 \pm 0.1$ & ns \\
\hline \multirow[t]{5}{*}{ Dark period } & HR (bpm) & $539 \pm 6$ & $550 \pm 5$ & ns \\
\hline & $\mathrm{SBP}(\mathrm{mmHg})$ & $132.8 \pm 1.3$ & $130.5 \pm 1.6$ & ns \\
\hline & MAP (mmHg) & $120.2 \pm 1.2$ & $116.9 \pm 1.3$ & ns \\
\hline & DBP (mmHg) & $108.6 \pm 1.3$ & $103.5 \pm 1.4$ & 0.01 \\
\hline & Mean activity (a.u./30s) & $2.9 \pm 0.3$ & $2.6 \pm 0.2$ & ns \\
\hline
\end{tabular}

HR, heart rate; SBP, systolic blood pressure; MAP, mean arterial pressure; DBP, diastolic blood pressure; a.u., arbitrary units; ns, not significant. 
Table 2. Summary of basal hemodynamic values during active and inactive periods under $12 \mathrm{~h} / 12 \mathrm{~h}$ light-dark cycle in Per2 mutants and wild type control mice.

Period

24h values Inactive periods (\%)

Active periods (\%)

HR no activity (bpm)

HR activity (bpm)

SBP no activity (mmHg)

SBP activity $(\mathrm{mmHg})$

MAP no activity $(\mathrm{mmHg})$

MAP activity ( $\mathrm{mmHg})$

DBP no activity ( $\mathrm{mmHg}$ )

DBP activity (mmHg)

Inactive periods (\%)

Active periods (\%)

HR no activity (bpm)

HR activity (bpm)

SBP no activity (mmHg)

SBP activity (mmHg)

MAP no activity (mmHg)

MAP activity (mmHg)

DBP no activity $(\mathrm{mmHg})$

DBP activity (mmHg)
Wild type

$$
\text { (n=30) }
$$

$74.3 \pm 1.1$

$25.7 \pm 1.2$

$499 \pm 5$

$580 \pm 5$

$125.5 \pm 1.3$

$137.1 \pm 1.5$

$113.3 \pm 1.1$

$123.3 \pm 1.2$

$101.9 \pm 1.3$

$110.9 \pm 1.4$

$84.1 \pm 1.0$

$15.9 \pm 1.0$

$486 \pm 6$

$561 \pm 5$

$122.0 \pm 1.3$

$131.7 \pm 1.6$

$109.7 \pm 1.1$

$117.7 \pm 1.3$

$98.0 \pm 1.3$

$105.1 \pm 1.3$
Per2 mutants

$P$ value

$$
\text { (n=27) }
$$

$72.2 \pm 1.0$

ns

$27.8 \pm 1$

ns

$521 \pm 5$

0.007

$587 \pm 4$

ns

$124.0 \pm 1.7$

ns

$135.6 \pm 1.7$

ns

$111.5 \pm 1.3 \quad$ ns

$120.4 \pm 1.4$

ns

$98.5 \pm 1.4$

ns

$106.1 \pm 1.5$

0.02

$80.1 \pm 1.2$

0.01

$19.9 \pm 1.2$

0.01

$515 \pm 6$

0.001

$582 \pm 4$

0.002

$121.0 \pm 1.7$

ns

$132.9 \pm 1.8$

ns

$108.6 \pm 1.4 \quad$ ns

$117.1 \pm 1.5 \quad$ ns

$95.6 \pm 1.5$

ns

$103.1 \pm 1.6$

ns 


\begin{tabular}{|c|c|c|c|c|}
\hline \multirow[t]{10}{*}{ Dark period } & Inactive periods $(\%)$ & $65.2 \pm 1.6$ & $65.6 \pm 1.2$ & ns \\
\hline & Active periods $(\%)$ & $34.8 \pm 1.6$ & $34.4 \pm 1.2$ & ns \\
\hline & HR no activity (bpm) & $515 \pm 6$ & $528 \pm 5$ & ns \\
\hline & HR activity (bpm) & $587 \pm 5$ & $590 \pm 5$ & ns \\
\hline & SBP no activity (mmHg) & $129.3 \pm 1.4$ & $127.2 \pm 1.7$ & ns \\
\hline & SBP activity $(\mathrm{mmHg})$ & $139.0 \pm 1.5$ & $136.7 \pm 1.8$ & ns \\
\hline & MAP no activity $(\mathrm{mmHg})$ & $117.4 \pm 1.2$ & $114.6 \pm 1.3$ & ns \\
\hline & MAP activity (mmHg) & $125.5 \pm 1.3$ & $121.8 \pm 1.5$ & ns \\
\hline & DBP no activity ( $\mathrm{mmHg}$ ) & $106.2 \pm 1.3$ & $101.4 \pm 1.4$ & 0.01 \\
\hline & DBP activity (mmHg) & $113.1 \pm 1.4$ & $107.4 \pm 1.6$ & 0.01 \\
\hline
\end{tabular}

HR, heart rate; MAP, mean arterial pressure; \%, percentage; ns, not significant. 
Table 3. Circadian rhythm periods as assessed by a Cosinor periodogram over 2 days of 12h/12h light-dark cycle (LD) and over 8 days of constant darkness (DD) in Per2 mutants and wild type control mice

$\begin{array}{lll} & \text { WT mice } & \text { Per2 mutants } \\ \text { MAP (LD) } & 24.1 \pm 0.4 \mathrm{~h} & 24.5 \pm 0.3 \mathrm{~h} \S \\ \text { MAP (DD) } & 24.2 \pm 0.1 \mathrm{~h} & 23.3 \pm 0.1 \mathrm{~h} * * * \\ \text { HR (LD) } & 24.1 \pm 0.3 \mathrm{~h} & 24.6 \pm 0.5 \mathrm{~h} \S \\ \text { HR (DD) } & 24.1 \pm 0.04 \mathrm{~h} & 23.2 \pm 0.1 \mathrm{~h} * * * \\ \text { Activity (LD) } & 24.4 \pm 0.5 \mathrm{~h} & 24.6 \pm 0.7 \mathrm{~h} \\ \text { Activity (DD) } & 24.0 \pm 0.05 \mathrm{~h} & 23.3 \pm 0.2 \mathrm{~h} * * *\end{array}$

$\mathrm{HR}$, heart rate; MAP, mean arterial pressure.

WT mice: $\mathrm{n}=6$; Per2 mutants: $\mathrm{n}=9$ (except $\mathrm{n}=7$ for locomotor activity under DD)

LD vs. DD: $\S \mathrm{P}<0.05, \S \S \mathrm{P}<0.01$

Per2 mutant mice vs. WT mice: $* * * \mathrm{P}<0.001$ 


\section{Figure Legends}

Figure 1. Dark-light differences in mean arterial pressure (MAP, panel A), heart rate (HR, panel B) and locomotor activity (panel C) when plotted over $12 \mathrm{~h} / 12 \mathrm{~h}$ light-dark cycles ( $\mathrm{n}=30$ for wild type mice, $\mathrm{n}=27$ for Per2 mutant mice). $* * * \mathrm{P}<0.001$ (compared to wild type). Values for individual mice are plotted as separate symbols.

Figure 2. Circadian rhythm periods as assessed by a chi square periodogram over the 8 consecutive days of constant darkness for mean arterial pressure (MAP, panel A), heart rate (HR, panel B) and locomotor activity (panel C). ( $n=6$ for wild type mice, $n=9$ for Per 2 mutant mice except $\mathrm{n}=7$ for locomotor activity). In two Per 2 mutant mice the periodogram for locomotor activity did not yield a period length due to arrhythmicity.) $* * \mathrm{P}<0.01 ; * * * \mathrm{P}<0.001$ (compared to wild type). Values for individual mice are plotted as separate symbols.

Figure 3. Left panels: "Night" - "day" differences in mean arterial pressure (MAP, panel A1), heart rate (HR, panel B1) and locomotor activity (panel C1) when plotted over 12h/12h cycles (Zeitgeber time, ZT) before, during and after of 8 days of constant darkness (DD). LD = light-dark cycle. Right panels: "Subjective night"-“subjective day" difference obtain from the same data based on recalculated individual circadian periods (Circadian time, CT). $(\mathrm{n}=6$ for wild type mice, $\mathrm{n}=9$ for Per2 mutant mice except $\mathrm{n}=7$ for locomotor activity under constant darkness). $* \mathrm{P}<0.05 ; \S \mathrm{P}<0.001$ (compared to control LD baseline values)

Figure 4. Acrophase and threshold of circadian rhythm for mean arterial pressure (MAP, panel A), heart rate (HR, panel B) and locomotor activity (panel C), for control light- 
dark cycle (LD) and for each individual of 8 days of constant darkness (DD). ( $n=6$ for wild type mice, $\mathrm{n}=9$ for Per2 mutant mice except $\mathrm{n}=7$ for locomotor activity under DD). $\S \mathrm{P}<0.05$ compared to control LD baseline values;

* $\mathrm{P}<0.05$ compared to wild type mice. 
A

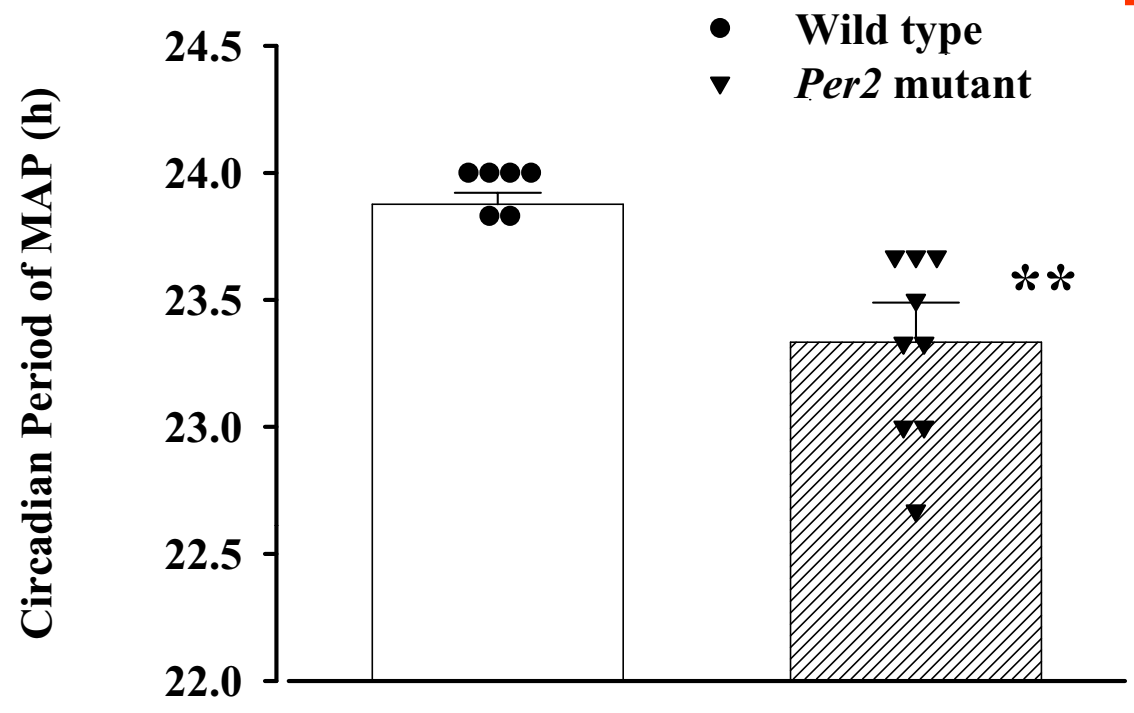

B

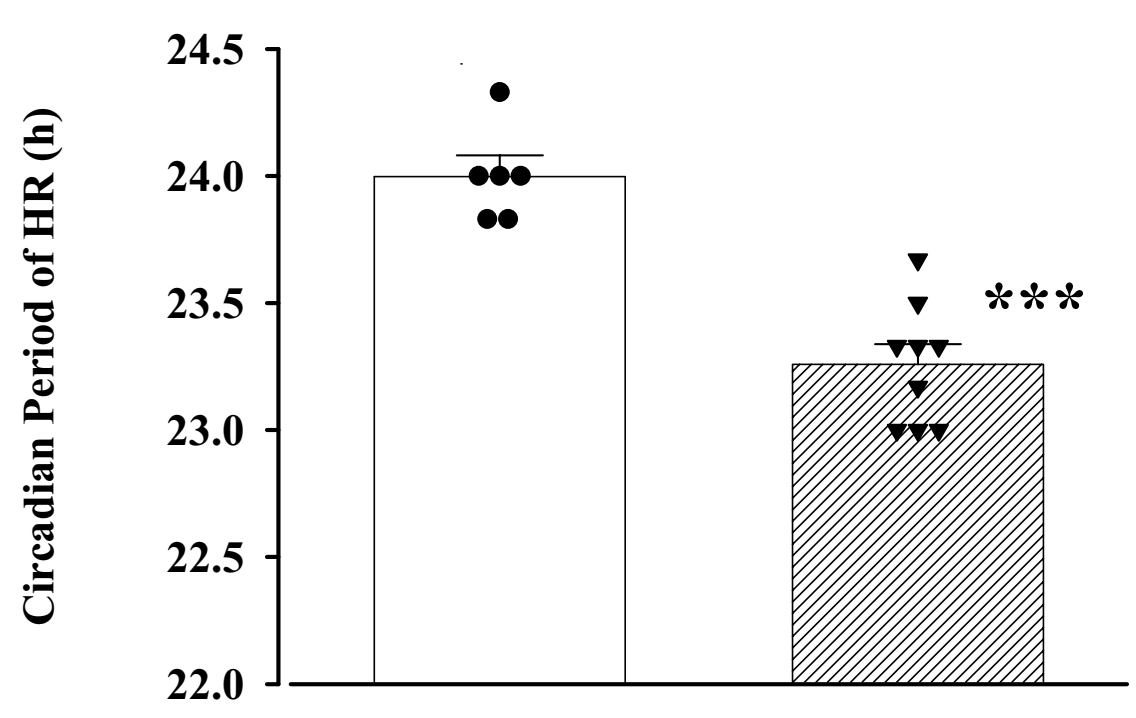

C

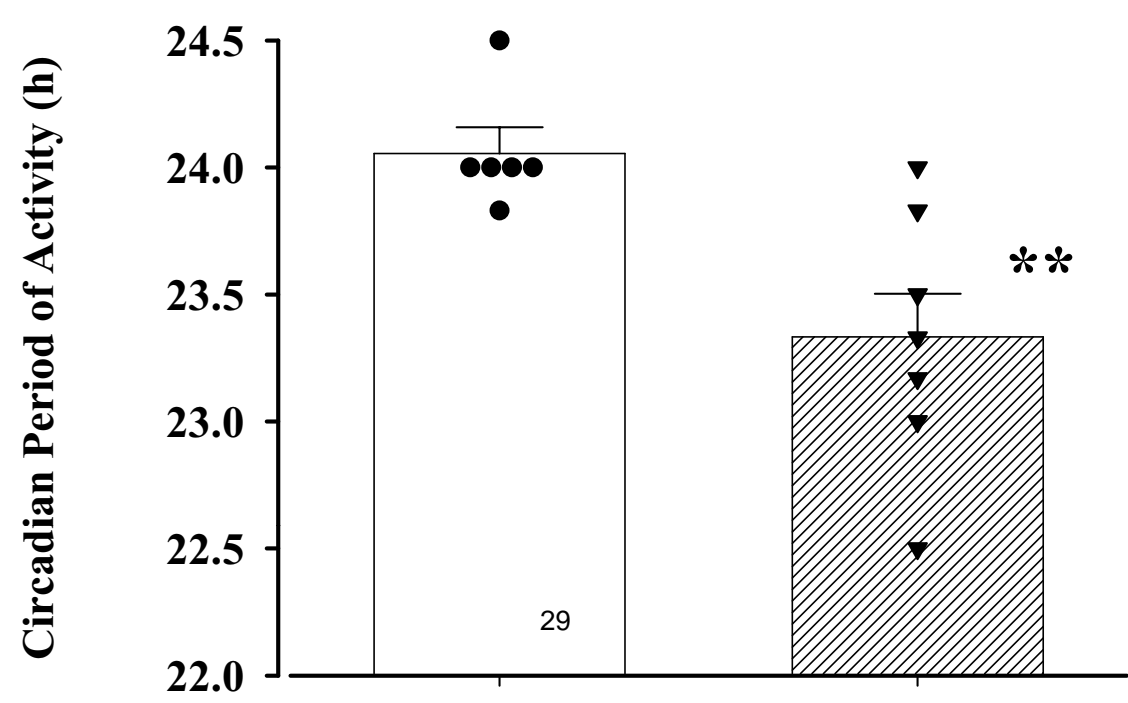

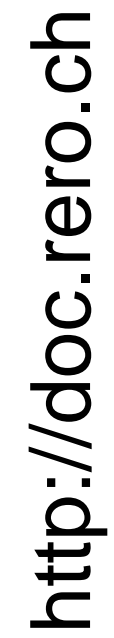


A
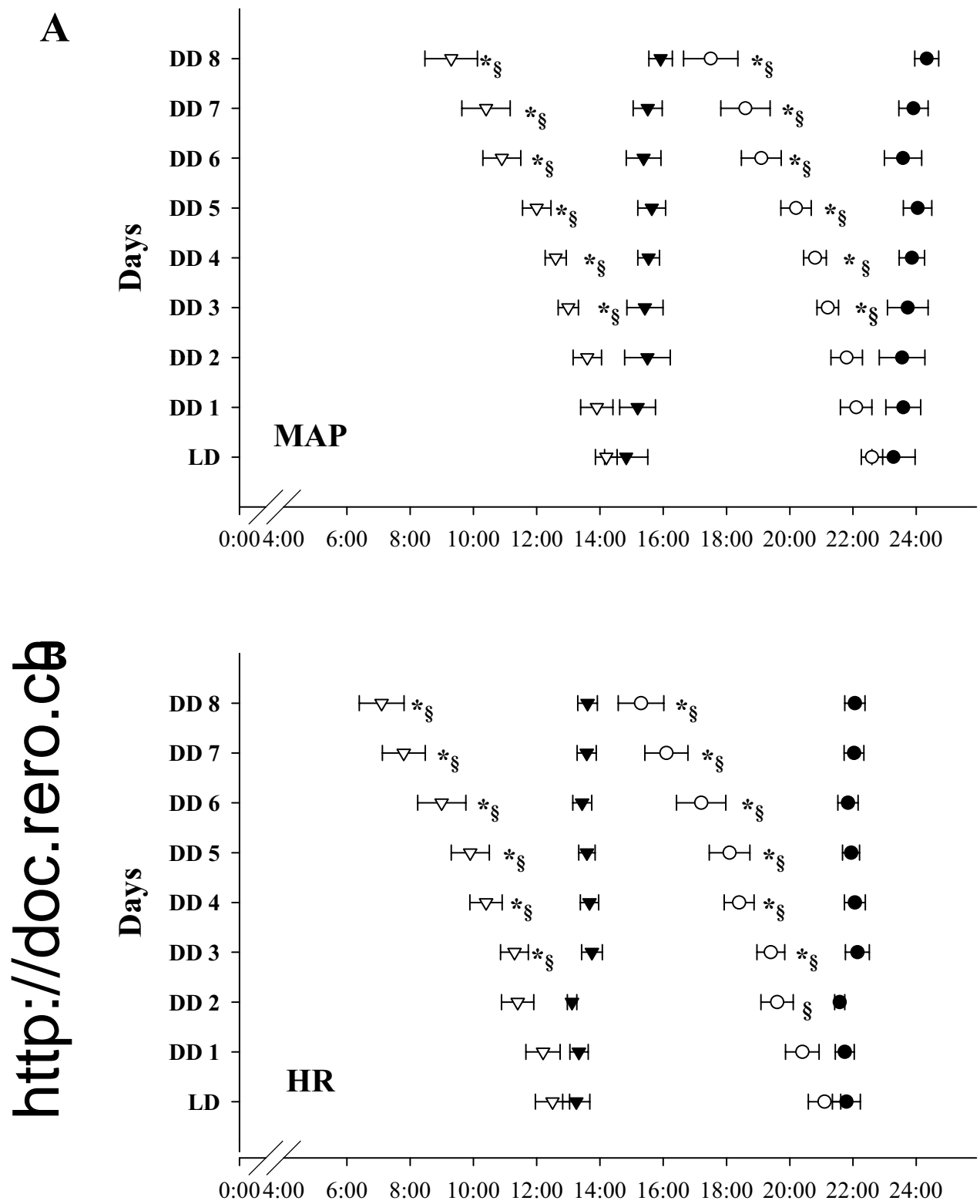

C

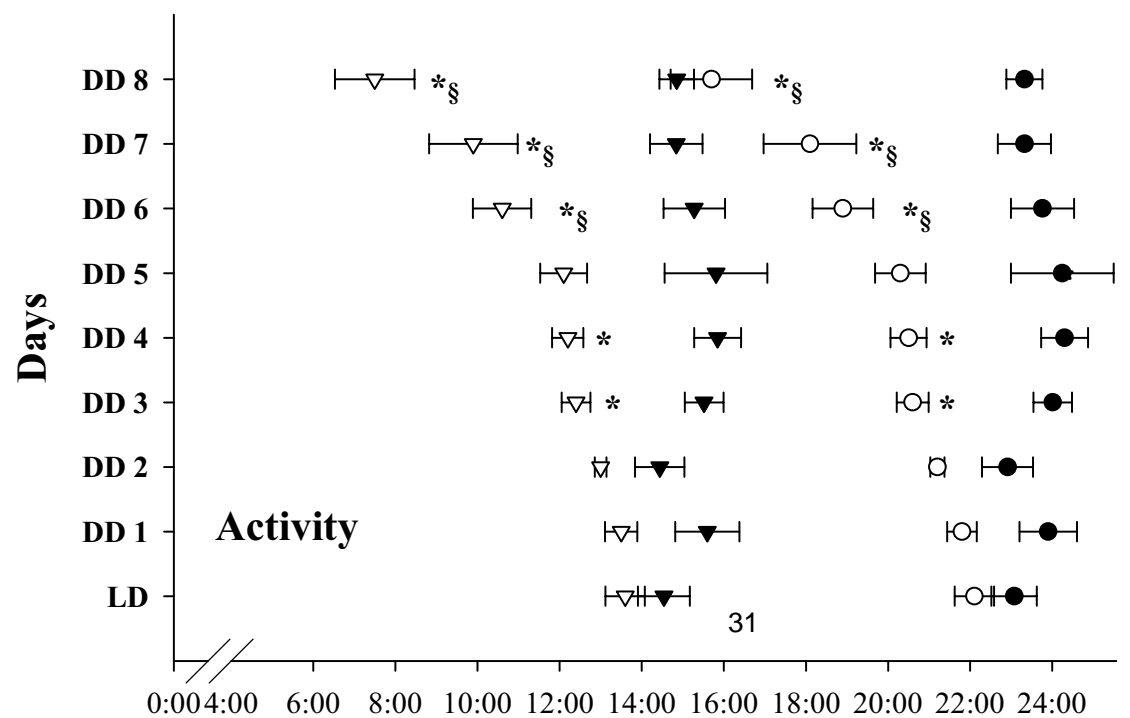

- WT acrophase 